\title{
FREDHOLM EIGENVALUE FOR A QUASI-CIRCLE AND GRUNSKY FUNCTIONALS
}

\author{
Yuliang Shen \\ Soochow University, Department of Mathematics \\ Suzhou 215006, P. R. China; ylshen@suda.edu.cn
}

\begin{abstract}
We give several new formulas of the least positive Fredholm eigenvalue for a quasicircle, answering a problem posed recently by Kühnau. During the proof, we show that the Grunsky functionals corresponding to the two complementary domains of the quasi-circle are the same and equal to the reciprocal of the Fredholm eigenvalue.
\end{abstract}

\section{Introduction}

Let $\Gamma$ be a Jordan curve in the extended complex plane $\hat{\mathbf{C}}$ with complementary domains $D$ and $D^{*}$. Then the least positive Fredholm eigenvalue is the curve functional $\lambda_{\Gamma}$ defined by the equality (see Schiffer [14])

$$
\frac{1}{\lambda_{\Gamma}}=\sup _{\mathscr{H}} \frac{\left|\mathscr{D}_{D}\left[h_{1}\right]-\mathscr{D}_{D^{*}}\left[h_{2}\right]\right|}{\mathscr{D}_{D}\left[h_{1}\right]+\mathscr{D}_{D^{*}}\left[h_{2}\right]}=\frac{\max \left\{s\left(D, D^{*}\right), s\left(D^{*}, D\right)\right\}-1}{\max \left\{s\left(D, D^{*}\right), s\left(D^{*}, D\right)\right\}+1} .
$$

Here and in what follows,

$$
\mathscr{D}_{\Omega}[h]=\frac{1}{\pi} \iint_{\Omega}\left(\left|\partial_{z} h\right|^{2}+\left|\partial_{\bar{z}} h\right|^{2}\right) d x d y
$$

is the Dirichlet integral, $\mathscr{H}$ is the set of all the pairs $\left(h_{1}, h_{2}\right)$, where $h_{1}$ is real-valued and continuous in $\bar{D}$, harmonic in $D$, while $h_{2}$ is real-valued and continuous in $\overline{D^{*}}$, harmonic in $D^{*}$, such that $h_{1}=h_{2}$ on $\Gamma$, and $\mathscr{D}_{D}[h]+\mathscr{D}_{D^{*}}[h]<+\infty$. Also

$$
s\left(D, D^{*}\right)=\sup _{\mathscr{H}} \frac{\mathscr{D}_{D}\left[h_{1}\right]}{\mathscr{D}_{D^{*}}\left[h_{2}\right]}, \quad s\left(D^{*}, D\right)=\sup _{\mathscr{H}} \frac{\mathscr{D}_{D^{*}}\left[h_{2}\right]}{\mathscr{D}_{D}\left[h_{1}\right]}
$$

are known as the Schober's domain functionals (see Schober [15]). Clearly, $\lambda_{\Gamma} \geq 1$, and $\lambda_{\Gamma}=+\infty$ if and only if $\Gamma$ is a circle, while $\lambda_{\Gamma}>1$ if and only if $\Gamma$ is a quasi-circle, namely, $\Gamma$ is the image of the unit circle under some quasiconformal mapping of the complex plane (see [1], [22]).

It is well known that the least positive Fredholm eigenvalue $\lambda_{\Gamma}$ is very important to the study in geometric function theory, in particular, it plays a significant role in determining the rate of convergence of the classical Neumann-Poincaré series (see [2], [13]), and many interesting properties of $\lambda_{\Gamma}$ have been obtained in the literature (see [1], [7], [13-16], [22]). We recall the following fact (see [18-19] and [10]) which

doi:10.5186/aasfm.2010.3536

2000 Mathematics Subject Classification: Primary 30C62; Secondary 30C55, 47B37.

Key words: Fredholm eigenvalue, quasi-circle, Grunsky functional.

Research supported by Program for New Century Excellent Talents in University and the National Natural Science Foundation of China. 
will be used later: for any quasi-circle $\Gamma, s\left(D, D^{*}\right)=s\left(D^{*}, D\right)$, so Schober's domain functionals $s$ are actually curve functionals, and

$$
\frac{\lambda_{\Gamma}+1}{\lambda_{\Gamma}-1}=\sup _{\mathscr{H}} \frac{\mathscr{D}_{D}\left[h_{1}\right]}{\mathscr{D}_{D^{*}}\left[h_{2}\right]}=\sup _{\mathscr{H}} \frac{\mathscr{D}_{D^{*}}\left[h_{2}\right]}{\mathscr{D}_{D}\left[h_{1}\right]} .
$$

When $\Gamma$ is three times continuously differentiable, this fact was already pointed out by Schober [15, p. 379].

Recently, some new characterizations of the Fredholm eigenvalue $\lambda_{\Gamma}$ were given by Kühnau [8-11] (see also [21]). In particular, the following result was proved in [8] and [11].

Theorem A. Let $\Gamma$ be a piecewise analytic Jordan curve. Then it holds that

$$
\frac{\lambda_{\Gamma}+1}{\lambda_{\Gamma}-1}=\sup \frac{\left|\int_{\Gamma} h_{1} d h_{2}\right|^{2}}{4 \mathscr{D}_{D}\left[h_{1}\right] \mathscr{D}_{D^{*}}\left[h_{2}\right]},
$$

where the sup is taken from all functions $h_{1}$ and $h_{2}$ which are real analytic in $\bar{D}$ and $\overline{D^{*}}$ respectively.

As pointed out by Kühnau [11], a great advantage of the property (1.5) of $\lambda_{\Gamma}$ in comparison with (1.1), (1.3) and (1.4) is that the functions $h_{1}$ and $h_{2}$ are not necessarily linked at the curve $\Gamma$ : we can choose $h_{1}$ and $h_{2}$ completely independently. On the other hand, there is the disadvantage that in the formulation and the proof of (1.5), the Jordan curve $\Gamma$ is assumed to be piecewise analytic. There arises the natural question whether (1.5) still holds for a general quasi-circle $\Gamma$, which was also asked by Kühnau [11].

In this paper, we will extend Theorem $A$ in case of a general quasi-circle $\Gamma$ (see Theorem 5.1), which gives a positive answer to the above-mentioned problem. Our proof is based on univalent function theory and thus differs largely from Kühnau's discussion. Here is the organization of the paper: in section 2 we recall some basic results on univalent functions, especially on Grunsky inequalities; in sections 3 and 4 we shall study the (generalized) Faber polynomials for univalent functions in the exterior of the unit disk along the lines in the paper [20], where we have dealt with the Faber polynomials for univalent functions in the unit disk; we will give the proof of our results in the final section 5, and as a by-product, we show that the Grunsky functionals corresponding to the two complementary domains of the quasi-circle $\Gamma$ are the same and equal to the reciprocal of the Fredholm eigenvalue $\lambda_{\Gamma}$, a fact which appears to have gone unnoticed.

\section{Grunsky functionals}

We begin with some well known results from univalent function theory (see [12], and also [5]). For a meromorphic function $g(z)=b z+\sum_{n=0}^{+\infty} b_{n} z^{-n}, b \neq 0$, in a neighborhood of $\infty$ in the extended complex plane $\hat{\mathbf{C}}$, its Grunsky coefficients $\beta_{m n}(g)$ are determined from the expression

$$
\log \frac{g(z)-g(\zeta)}{z-\zeta}=\log b-\sum_{m, n=1}^{+\infty} \beta_{m n}(g) z^{-m} \zeta^{-n}
$$


Then $g$ can be extended to a univalent function in $\Delta^{*}=\{z:|z|>1\} \cup\{\infty\}$ and belongs to the class $\tilde{\Sigma}$ if and only if the inequality

$$
\left|\sum_{m, n=1}^{+\infty} \sqrt{m n} \beta_{m n}(g) x_{m} x_{n}\right| \leq \sum_{m=1}^{+\infty}\left|x_{m}\right|^{2}
$$

holds for any $x=\left(x_{1}, x_{2}, \cdots\right) \in l^{2}$. If $g \in \tilde{\Sigma}$ can be extended to a $k$-quasiconformal mapping inside the unit disk $\Delta=\{z:|z|<1\}$ and belongs to the subclass $\tilde{\Sigma}(k)$, its Grunsky coefficients $\beta_{m n}(g)$ satisfy the stronger inequality

$$
\left|\sum_{m, n=1}^{+\infty} \sqrt{m n} \beta_{m n}(g) x_{m} x_{n}\right| \leq k \sum_{m=1}^{+\infty}\left|x_{m}\right|^{2} .
$$

Conversely, from the inequality (2.3) we can deduce that $g$ can be extended to a $k^{\prime}$-quasiconformal mapping into $\Delta$ with $k^{\prime} \geq k$, namely, $g \in \bigcup_{k<1} \tilde{\Sigma}(k)$.

It was an open question whether the (necessary) condition (2.3) is also sufficient for $g \in \tilde{\Sigma}(k)$ before Kühnau [6] gave some concrete example which says that there exists $g \in \tilde{\Sigma}$ which satisfies (2.3) but can not be extended to a $k^{\prime}$-quasiconformal mapping inside the unit disk $\Delta$ with $k^{\prime} \leq k$. By means of the extremal dilatation

$$
k(g)=\min \left\{k: g \in \cup_{k<1} \tilde{\Sigma}(k)\right\},
$$

and the Grunsky functional

$$
b(g)=\sup _{x \in S l^{2}}\left|\sum_{m, n=1}^{+\infty} \sqrt{m n} \beta_{m n}(g) x_{m} x_{n}\right|,
$$

where $S l^{2}$ is the unit sphere in $l^{2}$, Krushkal [3-4] gave a complete solution to the question.

Similarly, we denote by $\tilde{S}$ the class of all univalent functions $f(z)=\sum_{n=1}^{+\infty} a_{n} z^{n}$ in $\Delta$, and by $\tilde{S}(k)$ the subclass of $\tilde{S}$ consisting of those functions which can be extended to a $k$-quasiconformal mapping to $\Delta^{*}$. For each function $f \in \tilde{S}$, we may define its Grunsky coefficients $\alpha_{m n}(f)$, extremal dilatation $k(f)$ and Grunsky functional $b(f)$ in the same manner as (2.1), (2.4) and (2.5). Specifically, $\alpha_{m n}(f)$ are determined from the expression

$$
\log \frac{f(z)-f(\zeta)}{z-\zeta}=-\sum_{m, n=0}^{+\infty} \alpha_{m n}(f) z^{m} \zeta^{n} .
$$

Now let $\Gamma$ be a quasi-circle in the extended complex plane $\hat{\mathbf{C}}$ with complementary domains $D$ and $D^{*}$ such that $0 \in D$ and $\infty \in D^{*}$. Then there exists a uniquely determined pair of functions $f$ and $g$ in $\bigcup_{k<1} \tilde{S}(k)$ and $\cup_{k<1} \tilde{\Sigma}(k)$ respectively, such that $f^{\prime}(0)>0, g^{\prime}(\infty)>0$, and $f(\Delta)=D, g\left(\Delta^{*}\right)=D^{*}$. It is easy to see that $k(f)=k(g)$ for each quasi-circle $\Gamma$. As we shall see in section 5 , the following analogous result holds.

Theorem 2.1. Under the notations above, it holds that $b(f)=b(g)$.

We end this section with some background on the Fredholm eigenvalue and Grunsky functionals. By the quasi-invariance of Dirichlet integral under quasiconformal mappings, Ahlfors [1] observed that $\lambda_{\Gamma}^{-1} \leq k(f)(=k(g))$. It is also known that the Fredholm eigenvalue $\lambda_{\Gamma}$ and Grunsky functionals are closely related to each other. 
In an important article [6], Kühnau proved that $b(g)=\lambda_{\Gamma}^{-1}$ when $\Gamma$ is smooth. Actually, this fact is implicit in the fundamental work of Bergman and Schiffer (see [2], [14]). By means of this result, Kühnau [6] found the first example of a univalent function $g \in \bigcup_{k<1} \tilde{\Sigma}(k)$ with $b(g)<k(g)$. Later, Kühnau [7] was able to prove that $b(g)=\lambda_{\Gamma}^{-1}$ for any quasi-circle $\Gamma$ by an approximation process. In section 5 , we will prove the following result, form which and (1.4) Theorem 2.1 follows immediately. It also provides a somewhat different approach to Kühnau's result that $b(g)=\lambda_{\Gamma}^{-1}$.

Theorem 2.2. Under the notations above, it holds that

$$
b(f)=\frac{s\left(D, D^{*}\right)-1}{s\left(D, D^{*}\right)+1}, \quad b(g)=\frac{s\left(D^{*}, D\right)-1}{s\left(D^{*}, D\right)+1} .
$$

\section{Convergence properties for Faber polynomials}

In the classical approach to the Grunsky inequalities (2.2), (2.3), Faber polynomials play an important role (see [12]). In a previous paper [20], the author obtained some fundamental properties of Faber polynomials for univalent functions in the class $\tilde{\Sigma}$, which as will be seen are crucial in the proof of our results. We need to explore some analogous properties of Faber polynomials for univalent functions in the class $\tilde{S}$. For the sake of completeness and also of their own interest, we will go into the details here.

Recall that the $n$-th (generalized) Faber polynomial $F_{n}(w)$ for $f \in \tilde{S}$ is a polynomial of $w^{-1}$ of degree $n$ determined by the following expression:

$$
\log \frac{w-f(z)}{w}=\log \frac{f(z)}{a_{1} z}-\sum_{n=1}^{+\infty} \frac{1}{n} F_{n}(w) z^{n}, \quad w \neq 0, z \rightarrow 0 .
$$

It follows from (2.6) and (3.1) that Grunsky coefficients and Faber polynomials are related by the following relation:

$$
F_{n}(f(z))=z^{-n}+n \sum_{m=1}^{+\infty} \alpha_{m n}(f) z^{m}, \quad 0 \neq z \in \Delta .
$$

By a standard computation, it follows that

$$
\frac{1}{\pi} \iint_{\hat{\mathbf{C}}-f(\Delta)}\left|F_{n}^{\prime}(w)\right|^{2} d u d v=n\left(1-n \sum_{m=1}^{+\infty} m\left|\alpha_{m n}(f)\right|^{2}\right) .
$$

More generally, it holds that

$$
\frac{1}{\pi} \iint_{\hat{\mathbf{C}}-f(\Delta)} F_{m}^{\prime}(w) \overline{F_{n}^{\prime}(w)} d u d v=\sqrt{m n}\left(\delta_{m, n}-\sqrt{m n} \sum_{j=1}^{+\infty} j \alpha_{m j}(f) \overline{\alpha_{n j}(f)}\right)
$$

where, as usual, $\delta_{m, n}=1$ when $m=n$, and $\delta_{m, n}=0$ when $m \neq n$.

Now set $D=f(\Delta)$. By Koebe's one-quarter theorem, $D$ contains the ball $\left\{w:|w| \leq\left|a_{1}\right| / 4\right\}$. We assume that $\hat{\mathbf{C}}-D$ has non-empty interior $D^{*}$ which contains a neighborhood of $\infty$. 
Note that (3.1) converges absolutely and locally uniformly in $D^{*} \times \Delta$. Differentiation of (3.1) with respect to $z$ gives

$$
\frac{f^{\prime}(z)}{f(z)}-\frac{1}{z}-\frac{f^{\prime}(z)}{f(z)-w}=\sum_{n=1}^{+\infty} F_{n}(w) z^{n-1}, \quad w \in D^{*}, z \in \Delta,
$$

which also converges absolutely and locally uniformly in $D^{*} \times \Delta$. Thus,

$$
\lim _{z \rightarrow 0}\left(\frac{f^{\prime}(z)}{f(z)}-\frac{1}{z}-\frac{f^{\prime}(z)}{f(z)-w}\right)=F_{1}(w)=\frac{a_{1}}{w}+\frac{a_{2}}{a_{1}} .
$$

Noting that $|w| \geq\left|a_{1}\right| / 4$ when $w \in D^{*}$, we conclude that there exist some $r<1$ and $M_{1}>0$ such that $\left|\frac{f^{\prime}(z)}{f(z)}-\frac{1}{z}-\frac{f^{\prime}(z)}{f(z)-w}\right|<M_{1}$ when $|z|<r$. Thus

$$
\iint_{|z|<r}\left|\frac{f^{\prime}(z)}{f(z)}-\frac{1}{z}-\frac{f^{\prime}(z)}{f(z)-w}\right|^{2} d x d y \leq M_{1}^{2} r^{2} \pi .
$$

Clearly, there exists some $R_{1}>0$ such that $|f(z)| \geq R_{1}$ when $|z| \geq r$. Since $D^{*}$ contains a neighborhood of $\infty$, there exists some $R_{2}>0$ such that $\left\{w:|w|>R_{2}\right\}$ is contained in $D^{*}$. Thus $|f(z)| \leq R_{2}$ whenever $z \in \Delta$. Now, for any $w \in D^{*}$, set $d\left(w, \partial D^{*}\right)=\inf \left\{|\zeta-w|: \zeta \in \partial D^{*}\right\}$. Then for any $z \in \Delta, d\left(w, \partial D^{*}\right) \leq|f(z)-w| \leq$ $2 R_{2}+d\left(w, \partial D^{*}\right)$. Thus,

$$
\begin{aligned}
& \iint_{r<|z|<1}\left|\frac{f^{\prime}(z)}{f(z)}-\frac{1}{z}-\frac{f^{\prime}(z)}{f(z)-w}\right|^{2} d x d y \\
& \leq 2 \iint_{r<|z|<1}\left(\left|\frac{f^{\prime}(z)}{f(z)}\right|^{2}+\frac{1}{|z|^{2}}+\left|\frac{f^{\prime}(z)}{f(z)-w}\right|^{2}\right) d x d y \\
& =2 \iint_{f(r<|z|<1)}\left(\frac{1}{|\zeta|^{2}}+\frac{1}{|\zeta-w|^{2}}\right) d \xi d \eta+4 \pi \log \frac{1}{r} \\
& \leq 2 \iint_{R_{1}<|\zeta|<R_{2}} \frac{1}{|\zeta|^{2}} d \xi d \eta+2 \iint_{d\left(w, \partial D^{*}\right)<|\zeta-w|<2 R_{2}+d\left(w, \partial D^{*}\right)} \frac{1}{|\zeta-w|^{2}} d \xi d \eta+4 \pi \log \frac{1}{r} \\
& =4 \pi \log \frac{R_{2}\left(2 R_{2}+d\left(w, \partial D^{*}\right)\right)}{R_{1} r d\left(w, \partial D^{*}\right)} .
\end{aligned}
$$

Consequently, there exist positive constants $M_{1}, M_{2}, M_{3}$ such that for any $w \in D^{*}$,

$$
\frac{1}{\pi} \iint_{\Delta}\left|\frac{f^{\prime}(z)}{f(z)}-\frac{1}{z}-\frac{f^{\prime}(z)}{f(z)-w}\right|^{2} d x d y \leq M_{1}+M_{2} \log \frac{M_{3}+d\left(w, \partial D^{*}\right)}{d\left(w, \partial D^{*}\right)} .
$$

It follows from (3.5) and (3.6) that

$$
\begin{aligned}
\sum_{n=1}^{+\infty} \frac{\left|F_{n}(w)\right|^{2}}{n} & =\frac{1}{\pi} \iint_{\Delta}\left|\frac{f^{\prime}(z)}{f(z)}-\frac{1}{z}-\frac{f^{\prime}(z)}{f(z)-w}\right|^{2} d x d y \\
& \leq M_{1}+M_{2} \log \frac{M_{3}+d\left(w, \partial D^{*}\right)}{d\left(w, \partial D^{*}\right)}
\end{aligned}
$$

We have proved

Proposition 3.1. $\sum_{n=1}^{+\infty} \frac{F_{n}^{2}}{n}$ converges absolutely and locally uniformly in $D^{*}$ and thus represents an analytic function in $D^{*}$. 
Remark 3.1. In general, $\sum_{n=1}^{+\infty} \frac{F_{n}^{2}}{n}$ does not converge absolutely on $\hat{\mathbf{C}}-D$ or uniformly in $D^{*}$. In fact, let $f$ be the identity map so that $D$ is the unit disk $\Delta$ and $\hat{\mathbf{C}}-D$ is the closed disk $\bar{\Delta}^{*}$. Then, $F_{n}(w)=w^{-n}$, and $\sum_{n=1}^{+\infty} \frac{F_{n}^{2}(w)}{n}=\sum_{n=1}^{+\infty} \frac{w^{-2 n}}{n}=$ $\log \frac{w^{2}}{w^{2}-1}$ converges neither absolutely on $\bar{\Delta}^{*}$ nor uniformly in $\Delta^{*}$. This also shows that the order in the inequality (3.7) is precise.

We proceed to derive another convergence property concerning the derivatives of Faber polynomials. Recall that the series in (3.5) converges absolutely and locally uniformly in $D^{*} \times \Delta$. For any natural number $p \geq 1$, differentiation of (3.5) with respect to $w \in D^{*} p$ times gives

$$
\frac{(-1)^{p} p ! f^{\prime}(z)}{(w-f(z))^{p+1}}=\sum_{n=1}^{+\infty} F_{n}^{(p)}(w) z^{-(n+1)}, \quad w \in D^{*}, z \in \Delta,
$$

which also converges absolutely and locally uniformly in $D^{*} \times \Delta$. By a similar reasoning as above, we conclude that there exist some constant $M_{p}$ such that for any $w \in D^{*}$,

$$
\sum_{n=1}^{+\infty} \frac{\left|F_{n}^{(p)}(w)\right|^{2}}{n}=\frac{p !}{\pi} \iint_{\Delta}\left|\frac{f^{\prime}(z)}{(w-f(z))^{p+1}}\right|^{2} d x d y \leq \frac{M_{p}}{d^{2 p}\left(w, \partial D^{*}\right)} .
$$

We have proved

Proposition 3.2. For any natural number $p \geq 1, \sum_{n=1}^{+\infty} \frac{\left(F_{n}^{(p)}\right)^{2}}{n}$ converges absolutely and locally uniformly in $D^{*}$ and represents an analytic function in $D^{*}$.

\section{Operators on $l^{2}$}

In this section, we shall discuss some important operators on the $l^{2}$ space, which will be used to prove our results in the next section. These results also have independent interest of their own.

Recall that $l^{2}$ is a Hilbert space of sequences $x=\left(x_{m}\right)$ with the inner product and norm

$$
\langle x, y\rangle=\sum_{m=1}^{+\infty} x_{m} \bar{y}_{m}, \quad\|x\|=\left(\sum_{m=1}^{+\infty}\left|x_{m}\right|^{2}\right)^{\frac{1}{2}} .
$$

Let $l_{0}^{2}$ denote the subspace of $l^{2}$ of sequences $x=\left(x_{m}\right)$ with all terms $x_{m}$, except possibly finitely many, being zero. Then $l_{0}^{2}$ is dense in $l^{2}$. In fact, for $x=\left(x_{1}, x_{2}, \cdots\right) \in l^{2}$, setting $x^{l}=\left(x_{1}, x_{2}, \cdots, x_{l}, 0,0, \cdots\right)$, then $x^{l} \in l_{0}^{2}$, and $x^{l} \rightarrow x$ as $l \rightarrow+\infty$.

Let $f \in \tilde{S}$ be given. Then $f \in \tilde{S}$ determines the so-called Grunsky operator $G(f): l^{2} \rightarrow l^{2}$ by

$$
G(f):\left(x_{m}\right) \mapsto\left(\sum_{n=1}^{+\infty} \sqrt{m n} \alpha_{m n}(f) x_{n}\right)
$$

so that

$$
\langle G(f) x, \bar{x}\rangle=\sum_{m, n=1}^{+\infty} \sqrt{m n} \alpha_{m n}(f) x_{m} x_{n}
$$


and

$$
\|G(f) x\|^{2}=\sum_{m=1}^{+\infty}\left|\sum_{n=1}^{+\infty} \sqrt{m n} \alpha_{m n}(f) x_{n}\right|^{2} .
$$

Since $\alpha_{m n}(f)=\alpha_{n m}(f)$, Schur's result ([17]) implies that

$$
b(f)=\sup _{x \in S l^{2}}|\langle G(f) x, \bar{x}\rangle|=\sup _{x \in S l^{2}}\|G(f) x\| .
$$

It follows from the results in section 2 that $G(f)$ is a bounded operator of norm $b(f) \leq 1$, and $b(f)<1$ if and only if $f \in \bigcup_{k<1} \tilde{S}(k)$.

We continue to use the notations in the last section, namely, $D=f(\Delta)$, and $D^{*}$ is the non-empty interior of $\hat{\mathbf{C}}-D$, which contains a neighborhood of $\infty$. Consider

$$
P_{x, l}(w)=\sum_{n=1}^{l} \frac{x_{n}}{\sqrt{n}} F_{n}(w), \quad x=\left(x_{1}, x_{2}, \cdots\right) \in l^{2} .
$$

$P_{x, l}(w)$ is a polynomial of $w^{-1}$ of degree at most $l$. It follows from (3.4) that

$$
\frac{1}{\pi} \iint_{\hat{\mathbf{C}}-D}\left|P_{x, l}^{\prime}(w)\right|^{2} d u d v=\left\|x^{l}\right\|^{2}-\left\|G(f) x^{l}\right\|^{2} .
$$

More generally, it holds that for $x, y \in l^{2}$,

$$
\frac{1}{\pi} \iint_{\hat{\mathbf{C}}-D} P_{x, l}^{\prime}(w) \overline{P_{y, l}^{\prime}(w)} d u d v=\left\langle x^{l}, y^{l}\right\rangle-\left\langle G(f) x^{l}, G(f) y^{l}\right\rangle .
$$

Noting that

$$
\left(\sum_{n=1}^{+\infty} \frac{\left|x_{n}\right|}{\sqrt{n}}\left|F_{n}(w)\right|\right)^{2} \leq \sum_{n=1}^{+\infty}\left|x_{n}\right|^{2} \sum_{n=1}^{+\infty} \frac{\left|F_{n}(w)\right|^{2}}{n},
$$

we conclude by Proposition 3.1 that the function

$$
P_{x}(w)=\sum_{n=1}^{+\infty} \frac{x_{n}}{\sqrt{n}} F_{n}(w)
$$

converges absolutely and locally uniformly in $D^{*}$ and represents an analytic function in $D^{*}$. Thus,

$$
P_{x}^{\prime}(w)=\sum_{n=1}^{+\infty} \frac{x_{n}}{\sqrt{n}} F_{n}^{\prime}(w)
$$

converges locally uniformly in $D^{*}$. In fact, it follows from Proposition 3.2 that (4.10) also converges absolutely in $D^{*}$. By Fatou's lemma and (4.7) we get that

$$
\begin{aligned}
\frac{1}{\pi} \iint_{D^{*}}\left|P_{x}^{\prime}(w)\right|^{2} d u d v & \leq \liminf _{l \rightarrow+\infty} \frac{1}{\pi} \iint_{D^{*}}\left|P_{x, l}^{\prime}(w)\right|^{2} d u d v \\
& \leq \liminf _{l \rightarrow+\infty} \frac{1}{\pi} \iint_{\hat{\mathbf{C}}-D}\left|P_{x, l}^{\prime}(w)\right|^{2} d u d v \\
& =\|x\|^{2}-\|G(f) x\|^{2}
\end{aligned}
$$


Now for any domain $\Omega$ in the extended complex plane $\hat{\mathbf{C}}$, let $\mathscr{A} D(\Omega)$ denote the Hilbert space of all holomorphic functions (up to constants) $\phi$ in $\Omega$ with the inner product and norm

$$
\langle\phi, \psi\rangle=\frac{1}{\pi} \iint_{\Omega} \phi^{\prime}(w) \overline{\psi^{\prime}(w)} d u d v, \quad\|\phi\|=\left(\frac{1}{\pi} \iint_{\Omega}\left|\phi^{\prime}(w)\right|^{2} d u d v\right)^{\frac{1}{2}}<+\infty .
$$

Coming back to our situation, it follows from (4.11) that (4.9) determines a linear operator $P: l^{2} \rightarrow \mathscr{A} D\left(D^{*}\right)$ sending $x \in l^{2}$ to $P_{x} \in \mathscr{A} D\left(D^{*}\right)$ which is a bounded and thus continuous operator with norm $\|P\| \leq 1$. Consequently, $P_{x, l}=P_{x^{l}} \rightarrow P_{x}$ as $l \rightarrow+\infty$, so $P_{x}(w)=\sum_{n=1}^{+\infty} \frac{x_{n}}{\sqrt{n}} F_{n}(w)$ converges in $\mathscr{A} D\left(D^{*}\right)$. In particular, the first inequality in (4.11) is actually an equality. When $\hat{\mathbf{C}}-\left(D \cup D^{*}\right)$ has measure zero, we conclude that

$$
\left\|P_{x}\right\|^{2}=\|x\|^{2}-\|G(f) x\|^{2}, \quad x \in l^{2} .
$$

More generally, taking limit in (4.8) we obtain

$$
\left\langle P_{x}, P_{y}\right\rangle=\langle x, y\rangle-\langle G(f) x, G(f) y\rangle, \quad x, y \in l^{2} .
$$

The following result can be proved by the same reasoning as in [20]. It says that $f \in \tilde{S}$ belongs to $\bigcup_{k<1} \tilde{S}(k)$ if and only if each element of $\mathscr{A} D\left(D^{*}\right)$ can be uniquely represented as $\sum_{n=1}^{+\infty} \frac{x_{n}}{\sqrt{n}} F_{n}, x \in l^{2}$, which converges absolutely and locally uniformly in $D^{*}$ and converges in $\mathscr{A} D\left(D^{*}\right)$.

Proposition 4.1. $P: l^{2} \rightarrow \mathscr{A} D\left(D^{*}\right)$ is one to one and surjective if and only if $f$ can be extended to a quasiconformal map into the whole plane $\hat{\mathbf{C}}$, namely, $f \in \bigcup_{k<1} \tilde{S}(k)$.

\section{Main results}

In this section, we shall extend Theorem A to a general quasi-circle $\Gamma$ (see Theorem 5.1). During the proof, we shall obtain several formulas of the Fredholm eigenvalue $\lambda_{\Gamma}$. Recall that $\Gamma$ is a quasi-circle in the extended complex plane $\hat{\mathbf{C}}$ with complementary domains $D$ and $D^{*}$. Without loss of generality, we assume that $0 \in D$ and $\infty \in D^{*}$. Let $f$ and $g$ belong to $\bigcup_{k<1} \tilde{S}(k)$ and $\bigcup_{k<1} \tilde{\Sigma}(k)$ respectively, such that $f^{\prime}(0)>0, g^{\prime}(\infty)>0$, and $f(\Delta)=D, g\left(\Delta^{*}\right)=D^{*}$.

Denote by $\tilde{\mathscr{H}}$ the space of all the pairs $\left(h_{1}, h_{2}\right)$, where $h_{1}$ and $h_{2}$ are real-valued and harmonic in $D$ and $D^{*}$, respectively, with norm

$$
\left\|\left(h_{1}, h_{2}\right)\right\|=\left(\mathscr{D}_{D}\left[h_{1}\right]+\mathscr{D}_{D^{*}}\left[h_{2}\right]\right)^{\frac{1}{2}}<+\infty .
$$

It is well known that for each pair $\left(h_{1}, h_{2}\right)$ in $\tilde{\mathscr{H}}, h_{1}$ and $h_{2}$ have boundary values almost everywhere in $\Gamma=\partial D=\partial D^{*}$. We also denote these boundary values by $h_{1}$ and $h_{2}$, respectively. We denote by $\hat{\mathscr{H}}$ the subspace of $\tilde{\mathscr{H}}$ which consists of those pairs $\left(h_{1}, h_{2}\right)$ such that $h_{1}$ and $h_{2}$ are equal to each other on $\Gamma$ almost everywhere. Clearly, $\mathscr{H}$ can be considered as a sub-space of $\hat{\mathscr{H}}$. In the following, we sometimes use $h=h_{1} \chi_{D}+h_{2} \chi_{D^{*}}$, which is defined in $D \cup D^{*}$, to denote a pair $\left(h_{1}, h_{2}\right)$ in $\tilde{\mathscr{H}}$. Then, $\left.h\right|_{\bar{D}}=h_{1},\left.h\right|_{\overline{D^{*}}}=h_{2}$. We first prove the following result. 
Lemma 5.1. There exists some bounded linear isomorphism $\hat{P}: l^{2} \rightarrow \hat{\mathscr{H}}$ such that $\hat{P}_{x} \in \mathscr{H}$ if $x \in l_{0}^{2}$. Furthermore, for any $x \in l^{2}$, it holds that

$$
\mathscr{D}_{D}\left[\hat{P}_{x}\right]=\frac{1}{2}\left(\|x\|^{2}+2 \Re\langle G(f) x, \bar{x}\rangle+\|G(f) x\|^{2}\right), \mathscr{D}_{D^{*}}\left[\hat{P}_{x}\right]=\frac{1}{2}\left(\|x\|^{2}-\|G(f) x\|^{2}\right) .
$$

Proof. For any $x \in l^{2}$, set

$$
\tilde{P}_{x}(z)=\sum_{n=1}^{\infty} \frac{x_{n}}{\sqrt{n}} \bar{z}^{n}+\sum_{m, n=1}^{\infty} \sqrt{n} \alpha_{m n}(f) x_{n} z^{m}, \quad z \in \Delta .
$$

Clearly, the above series converges absolutely and locally uniformly in $\Delta$ and thus represents a harmonic function in $\Delta$. A direct computation will show

$$
\mathscr{D}_{\Delta}\left[\tilde{P}_{x}\right]=\|x\|^{2}+\|G(f) x\|^{2} .
$$

It follows from (5.4) that (5.3) determines a bounded linear operator $\tilde{P}$ on $l^{2}$ sending $x$ to $\tilde{P}_{x}$.

Define

$$
\hat{P}_{x}(w)= \begin{cases}\Re \tilde{P}_{x}\left(f^{-1}(w)\right), & w \in D ; \\ \Re P_{x}(w), & w \in D^{*}\end{cases}
$$

Clearly, $\hat{P}_{x}$ is real and harmonic in $D \cup D^{*}$. A direct computation will show that $\hat{P}_{x}$ satisfies (5.2). Consequently, (5.5) determines a bounded and thus a continuous linear operator $\hat{P}: l^{2} \rightarrow \tilde{\mathscr{H}}$ which sends $x$ to $\hat{P}_{x}$. Recall that $\hat{P}_{x}$ denotes the pair $\left(\Re \tilde{P}_{x}\left(f^{-1}\right), \Re P_{x}\right)$.

Let $x \in l_{0}^{2}$. Then $P_{x}$ is a polynomial of $w^{-1}$ and thus analytic in $\hat{\mathbf{C}}-\{0\}$, so $P_{x} \circ f$ is continuous in $\bar{\Delta}-\{0\}$. It follows from (3.2), (4.9) and (5.3) that

$$
\tilde{P}_{x}(z)=P_{x}(f(z))+\sum_{n=1}^{+\infty} \frac{x_{n}}{\sqrt{n}} \bar{z}^{n}-\sum_{n=1}^{+\infty} \frac{x_{n}}{\sqrt{n}} z^{-n}, \quad z \in \Delta .
$$

Thus, $\tilde{P}_{x}$ is continuous in $\bar{\Delta}$, and $\tilde{P}_{x}=P_{x} \circ f$ on the unit circle $\partial \Delta$. It follows that $\hat{P}_{x} \in \mathscr{H}$.

Now suppose $x \in l^{2}$. Set $x^{l}$ as before. Then $x^{l} \in l_{0}^{2}$, and $x^{l} \rightarrow x$ as $l \rightarrow+\infty$. By the continuity of the operators of $P$ and $\tilde{P}$, it follows that $P_{x^{l}} \rightarrow P_{x}, \tilde{P}_{x^{l}} \rightarrow \tilde{P}_{x}$. Noting that $P_{x^{l}} \circ f=\tilde{P}_{x^{l}}$ on $\partial \Delta$, we conclude that $\left.P_{x}\right|_{\partial D^{*}}$ and $\left.\tilde{P}_{x} \circ f^{-1}\right|_{\partial D}$ are equal to each other almost everywhere on $\Gamma$. Thus, $\hat{P}_{x} \in \hat{\mathscr{H}}$.

It is clear that $\hat{P}: l^{2} \rightarrow \hat{\mathscr{H}}$ is one-to-one. It remains to prove that $\hat{P}: l^{2} \rightarrow \hat{\mathscr{H}}$ is surjective. Let $h \in \hat{\mathscr{H}}$ be given. We choose an analytic function $F$ on $D^{*}$ such that $h=\Re F$ on $D^{*}$ so that $F \in \mathscr{A} D\left(D^{*}\right)$, and $\|F\|^{2}=2 \mathscr{D}_{D^{*}}[h]$. By Proposition 4.1, there exists some $x \in l^{2}$ such that $F=P_{x}$. Then $h=\Re P_{x}=\hat{P}_{x}$ on $D^{*}$. We continue to show that $\hat{P}_{x}=h$ on $D$. Actually, since $\left.h\right|_{\partial D}=\left.h\right|_{\partial D^{*}}=\left.\Re P_{x}\right|_{\partial D^{*}}=\left.\Re \tilde{P}_{x} \circ f^{-1}\right|_{\partial D}$ almost everywhere on $\Gamma$, we conclude by Dirichlet Principle that $h=\Re \tilde{P}_{x} \circ f^{-1}=\hat{P}_{x}$ on $D$.

Now we begin to prove Theorem 2.2. By Lemma 5.1, any $x \in l_{0}^{2}$ corresponds to $\hat{P}_{x} \in \mathscr{H}$ such that (5.2) holds. Thus

$$
\frac{\|x\|^{2}+2 \Re\langle G(f) x, \bar{x}\rangle+\|G(f) x\|^{2}}{\|x\|^{2}-\|G(f) x\|^{2}}=\frac{\mathscr{D}_{D}\left[\hat{P}_{x}\right]}{\mathscr{D}_{D^{*}}\left[\hat{P}_{x}\right]} \leq s\left(D, D^{*}\right) .
$$


Since $l_{0}^{2}$ is dense in $l^{2}$, we may choose a sequence $\left(x_{n}\right)$ in $l_{0}^{2}$ such that $\left\|x_{n}\right\|=1$, and $\Re\left\langle G(f) x_{n}, \bar{x}_{n}\right\rangle \rightarrow b(f)$ as $n \rightarrow+\infty$. Taking limit in $(5.7)$ we obtain $\frac{1+2 b(f)+b^{2}(f)}{1-b^{2}(f)} \leq$ $s\left(D, D^{*}\right)$, or equivalently, $\frac{1+b(f)}{1-b(f)} \leq s\left(D, D^{*}\right)$.

Conversely, for any $h \in \hat{\mathscr{H}}$, there exists some $x \in l^{2}$ such that $\hat{P}_{x}=h$. It follows from (5.2) that

$$
\frac{\mathscr{D}_{D}[h]}{\mathscr{D}_{D^{*}}[h]}=\frac{\mathscr{D}_{D}\left[\hat{P}_{x}\right]}{\mathscr{D}_{D^{*}}\left[\hat{P}_{x}\right]}=\frac{\|x\|^{2}+2 \Re\langle G(f) x, \bar{x}\rangle+\|G(f) x\|^{2}}{\|x\|^{2}-\|G(f) x\|^{2}} \leq \frac{1+b(f)}{1-b(f)} .
$$

Thus,

$$
s\left(D, D^{*}\right) \leq \sup _{\hat{\mathscr{H}}} \frac{\mathscr{D}_{D}[h]}{\mathscr{D}_{D^{*}}[h]} \leq \frac{1+b(f)}{1-b(f)} .
$$

This finishes the proof of the first part of Theorem 2.2.

The second part of Theorem 2.2 can be proved by the same way. For completeness, we sketch the proof here. We need some fundamental properties of Faber properties for $g$ which we have established in [20]. Precisely, let $G_{n}$ denote the $n$-th Faber polynomial for $g$, which is a polynomial of degree $n$ determined by the following expression:

$$
\log \frac{g(z)-w}{b z}=-\sum_{n=1}^{\infty} \frac{1}{n} G_{n}(w) z^{-n}, \quad w \in \mathbf{C}, z \rightarrow \infty .
$$

It follows from (2.1) and (5.8) that Grunsky coefficients and Faber polynomials are related by the following relation:

$$
G_{n}(g(z))=z^{n}+n \sum_{m=1}^{\infty} \beta_{m n}(g) z^{-m}, \quad z \in \Delta^{*} .
$$

It was proved in [20] that for any $x \in l^{2}$ the function

$$
Q_{x}(w)=\sum_{n=1}^{\infty} \frac{x_{n}}{\sqrt{n}} G_{n}(w)
$$

converges absolutely and locally uniformly in $D$ and also converges in $\mathscr{A} D(D)$. (5.10) then determines a bounded linear operator $Q: l^{2} \rightarrow \mathscr{A} D(D)$ which sends $x$ to $Q_{x}$. If we denote by $G(g): l^{2} \rightarrow l^{2}$ the Grunsky operator determined by

$$
G(g):\left(x_{m}\right) \mapsto\left(\sum_{n=1}^{+\infty} \sqrt{m n} \beta_{m n}(g) x_{n}\right),
$$

it then holds

$$
\left\|Q_{x}\right\|^{2}=\|x\|^{2}-\|G(g) x\|^{2}, \quad x \in l^{2} .
$$

For $x \in l^{2}$, set

$$
\tilde{Q}_{x}(z)=\sum_{n=1}^{\infty} \frac{x_{n}}{\sqrt{n}} \bar{z}^{-n}+\sum_{m, n=1}^{\infty} \sqrt{n} \beta_{m n}(g) x_{n} z^{-m}, \quad z \in \Delta^{*},
$$

and define

$$
\hat{Q}_{x}(w)= \begin{cases}\Re Q_{x}(w), & w \in D ; \\ \Re \tilde{Q}_{x}\left(g^{-1}(w)\right), & w \in D^{*}\end{cases}
$$


The second part of Theorem 2.2 follows from the following result.

Lemma 5.2. The operator $\hat{Q}$ which sends $x$ to $\hat{Q}_{x}$ is a bounded linear isomorphism from $l^{2}$ onto $\hat{\mathscr{H}}$ such that $\hat{Q}_{x} \in \mathscr{H}$ if $x \in l_{0}^{2}$. Furthermore, for any $x \in l^{2}$, it holds that

$$
\begin{aligned}
\mathscr{D}_{D}\left[\hat{Q}_{x}\right] & =\frac{1}{2}\left(\|x\|^{2}-\|G(g) x\|^{2}\right), \\
\mathscr{D}_{D^{*}}\left[\hat{Q}_{x}\right] & =\frac{1}{2}\left(\|x\|^{2}+2 \Re\langle G(g) x, \bar{x}\rangle+\|G(g) x\|^{2}\right) .
\end{aligned}
$$

Remark 5.1 From the above discussion, we conclude that in (1.1), (1.3) and (1.4) we may allow the functions taken from $\hat{\mathscr{H}}$, namely,

$$
\frac{\lambda_{\Gamma}+1}{\lambda_{\Gamma}-1}=\sup _{\hat{\mathscr{H}}} \frac{\mathscr{D}_{D}\left[h_{1}\right]}{\mathscr{D}_{D^{*}}\left[h_{2}\right]}=\sup _{\hat{\mathscr{H}}} \frac{\mathscr{D}_{D^{*}}\left[h_{2}\right]}{\mathscr{D}_{D}\left[h_{1}\right]} .
$$

On the other hand, we may impose some smooth condition on the functions in (1.4). Precisely, let $\mathscr{H}_{1}$ denote the set of pairs $\left(h_{1}, h_{2}\right)$ in $\mathscr{H}$ such that $h_{1}$ is harmonic in $\bar{D}$, and $\mathscr{H}_{2}$ the set of pairs $\left(h_{1}, h_{2}\right)$ in $\mathscr{H}$ such that $h_{2}$ is harmonic in $\overline{D^{*}}$. Then for any $x \in l^{2}, \hat{P}_{x} \in \mathscr{H}_{2}, \hat{Q}_{x} \in \mathscr{H}_{1}$. Thus, we have

$$
\frac{\lambda_{\Gamma}+1}{\lambda_{\Gamma}-1}=\sup _{\mathscr{H}_{1}} \frac{\mathscr{D}_{D^{*}}\left[h_{2}\right]}{\mathscr{D}_{D}\left[h_{1}\right]}=\sup _{\mathscr{H}_{2}} \frac{\mathscr{D}_{D}\left[h_{1}\right]}{\mathscr{D}_{D^{*}}\left[h_{2}\right]} .
$$

We proceed to extend Theorem A to a general quasi-circle. For any pair $\left(h_{1}, h_{2}\right) \in$ $\tilde{\mathscr{H}}$, we denote by $\tilde{h}_{2}$ the harmonic function in $D$ with boundary values $h_{2}$ on $\Gamma$, we also denote by $h_{1}^{*}$ and $\tilde{h}_{2}^{*}$ the harmonic conjugations of $h_{1}$ and $\tilde{h}_{2}$, respectively. In the following, we will use the notation

$$
\mathscr{D}_{\Omega}[u, v]=\frac{1}{\pi} \iint_{\Omega}\left(\partial_{z} u \partial_{\bar{z}} v+\partial_{\bar{z}} u \partial_{z} v\right) d x d y .
$$

It is easy to see that $\left|\mathscr{D}_{\Omega}[u, v]\right|^{2} \leq \mathscr{D}_{\Omega}[u] \mathscr{D}_{\Omega}[v]$. Then we have

Proposition 5.1. Under the above notations, it holds that

$$
\frac{\lambda_{\Gamma}+1}{\lambda_{\Gamma}-1}=\sup _{\tilde{\mathscr{H}}} \frac{\left|\mathscr{D}_{D}\left[h_{1}, \tilde{h}_{2}^{*}\right]\right|^{2}}{\mathscr{D}_{D}\left[h_{1}\right] \mathscr{D}_{D^{*}}\left[h_{2}\right]} .
$$

Proof. For any pair $\left(h_{1}, h_{2}\right) \in \tilde{\mathscr{H}}$, noting that $\left(\tilde{h}_{2}, h_{2}\right) \in \hat{\mathscr{H}}$, we conclude from (5.16) that

$$
\left|\mathscr{D}_{D}\left[h_{1}, \tilde{h}_{2}^{*}\right]\right|^{2} \leq \mathscr{D}_{D}\left[h_{1}\right] \mathscr{D}_{D}\left[\tilde{h}_{2}^{*}\right]=\mathscr{D}_{D}\left[h_{1}\right] \mathscr{D}_{D}\left[\tilde{h}_{2}\right] \leq \frac{\lambda_{\Gamma}+1}{\lambda_{\Gamma}-1} \mathscr{D}_{D}\left[h_{1}\right] \mathscr{D}_{D^{*}}\left[h_{2}\right] .
$$

Thus,

$$
\sup _{\tilde{\mathscr{H}}} \frac{\left|\mathscr{D}_{D}\left[h_{1}, \tilde{h}_{2}^{*}\right]\right|^{2}}{\mathscr{D}_{D}\left[h_{1}\right] \mathscr{D}_{D^{*}}\left[h_{2}\right]} \leq \frac{\lambda_{\Gamma}+1}{\lambda_{\Gamma}-1}
$$

Conversely, let $\left(h_{1}, h_{2}\right) \in \mathscr{H}_{2}$. Then, $h_{1}=\tilde{h}_{2}$. Noting that $\left(h_{1}^{*}, h_{2}\right)$ is a pair in $\tilde{\mathscr{H}}$, we conclude from (5.17) that

$$
\sup _{\tilde{\mathscr{H}}} \frac{\left|\mathscr{D}_{D}\left[h_{1}, \tilde{h}_{2}^{*}\right]\right|^{2}}{\mathscr{D}_{D}\left[h_{1}\right] \mathscr{D}_{D^{*}}\left[h_{2}\right]} \geq \sup _{\mathscr{H}_{2}} \frac{\left|\mathscr{D}_{D}\left[h_{1}^{*}, \tilde{h}_{2}^{*}\right]\right|^{2}}{\mathscr{D}_{D}\left[h_{1}^{*}\right] \mathscr{D}_{D^{*}}\left[h_{2}\right]}=\sup _{\mathscr{H}_{2}} \frac{\mathscr{D}_{D}\left[h_{1}\right]}{\mathscr{D}_{D^{*}}\left[h_{2}\right]}=\frac{\lambda_{\Gamma}+1}{\lambda_{\Gamma}-1} .
$$

This finishes the proof of Proposition 5.1. 
Let $\tilde{\mathscr{H}}_{2}$ denote the subspace of $\tilde{\mathscr{H}}$ consisting of the pairs $h=\left(h_{1}, h_{2}\right)$ such that $h_{1}$ is continuous in $\bar{D}$, harmonic in $D$, while $h_{2}$ is harmonic in $\overline{D^{*}}$. Note that we don't require that $h_{1}$ and $h_{2}$ have the same boundary values on $\Gamma$. Clearly, $\mathscr{H}_{2}$ can be considered as a subspace of $\tilde{\mathscr{H}}_{2}$. Examining the proof of Proposition 5.1 (and also of (5.17)), we find it holds that

$$
\frac{\lambda_{\Gamma}+1}{\lambda_{\Gamma}-1}=\sup _{\tilde{\mathscr{H}}_{2}} \frac{\left|\mathscr{D}_{D}\left[h_{1}, \tilde{h}_{2}^{*}\right]\right|^{2}}{\mathscr{D}_{D}\left[h_{1}\right] \mathscr{D}_{D^{*}}\left[h_{2}\right]} .
$$

On the other hand, if $h=\left(h_{1}, h_{2}\right) \in \tilde{\mathscr{H}}_{2}$, then $\int_{\Gamma} h_{1} d h_{2}$ is well defined, and as observed by Kühnau [11], we have

$$
\begin{aligned}
\int_{\Gamma} h_{1} d h_{2} & =\int_{\Gamma} h_{1} d \tilde{h}_{2}=\int_{\Gamma}\left(h_{1} \partial_{z} \tilde{h}_{2} d z+h_{1} \partial_{\bar{z}} \tilde{h}_{2} d \bar{z}\right) \\
& =2 i \iint_{D}\left(\partial_{\bar{z}}\left(h_{1} \partial_{z} \tilde{h}_{2}\right)-\partial_{z}\left(h_{1} \partial_{\bar{z}} \tilde{h}_{2}\right)\right) d x d y \\
& =2 i \iint_{D}\left(\partial_{\bar{z}} h_{1} \partial_{z} \tilde{h}_{2}-\partial_{z} h_{1} \partial_{\bar{z}} \tilde{h}_{2}\right) d x d y \\
& =-2 \iint_{D}\left(\partial_{\bar{z}} h_{1} \partial_{z} \tilde{h}_{2}^{*}+\partial_{z} h_{1} \partial_{\bar{z}} \tilde{h}_{2}^{*}\right) d x d y \\
& =-2 \mathscr{D}_{D}\left[h_{1}, \tilde{h}_{2}^{*}\right] .
\end{aligned}
$$

The following result follows from (5.19) and (5.20). It is a generalization of Theorem $\mathrm{A}$ in case of a general quasi-circle.

Theorem 5.1. Let $\Gamma$ be a quasi-circle with complementary domains $D$ and $D^{*}$. Then it holds that

$$
\frac{\lambda_{\Gamma}+1}{\lambda_{\Gamma}-1}=\sup \frac{\left|\int_{\Gamma} h_{1} d h_{2}\right|^{2}}{4 \mathscr{D}_{D}\left[h_{1}\right] \mathscr{D}_{D^{*}}\left[h_{2}\right]},
$$

where the sup is taken from all pairs $\left(h_{1}, h_{2}\right)$, where $h_{1}$ is continuous in $\bar{D}$, harmonic in $D$, while $h_{2}$ is harmonic in $\overline{D^{*}}$.

Acknowledgements. I wish to thank Professor Kühnau for sending me a series of papers. I also wish to express my gratitude to the referee for his useful advice.

\section{References}

[1] Ahlfors, L. V. : Remarks on the Neumann-Poincaré integral equations. - Pacific J. Math. 2, $1952,271-280$.

[2] Bergman, S., and M. M. Schiffer: Kernel functions and conformal mappings. - Compos. Math. 8, 1951, 205-249.

[3] Krushkal, S. L.: The Grunsky coefficient conditions. - Siberian Math. J. 28, 1987, 104-110.

[4] Krushkal, S. L.: Grunsky coefficient inequalities, Carathéodory metric and extremal quasiconformal mappings. - Comment. Math. Helv. 64, 1989, 650-660.

[5] KÜHNAU, R.: Verzerrungssätze und Koeffizientenbedingungen vom Grunskyschen Typ für quasi-konforme Abbildungen. - Math. Nachr. 48, 1971, 77-105.

[6] KÜhnau, R.: Zu den Grunskyschen Koeffizientenbedingungen .- Ann. Acad. Sci. Fenn. Ser. A I Math. 6, 1981, 125-130. 
[7] KÜHnau, R.: Quasikonforme Fortsetzbarkeit, Fredholmschen Eigenwerte und Grunskysche Koeffizientenbedingungen. - Ann. Acad. Sci. Fenn. Ser. A I Math. 7, 1982, 383-391.

[8] KÜHnaU, R.: Koeffizientenbedingungen vom Grunskyschen Typ und Fredholmsche Eigenwerte. - Ann. Univ. Mariae Curie-Skłodowska Lublin Sect. A 58, 2004, 79-87.

[9] KÜHNAU, R.: New characterizations of Fredholm eigenvalues of quasicircles. - Rev. Roumaine Math. Pure Appl. 51, 2006, 295-307.

[10] KÜHNAU, R.: A new matrix characterization of Fredholm eigenvalues of quasicircles. - J. Anal. Math. 99, 2006, 295-307.

[11] KÜHNAU, R.: Quadratic forms in geometric function theory, quasiconformal extensions, Fredholm eigenvalues. - Contemp. Math. 455, 2008, 237-256.

[12] Pommerenke, Сh.: Univalent Functions. - Vandenhoeck and Ruprecht, 1975.

[13] Schiffer, M.: Fredholm eigenvalues of plane domains. - Pacific J. Math. 7, 1957, 1187-1225.

[14] Schiffer, M.: Fredholm eigenvalues and Grunsky matrices. - Ann. Polon. Math. 39, 1981, $149-164$.

[15] Schober, G.: Continuity of curve functionals and a technique involving quasiconformal mappings. - Arch. Ration. Mech. Anal. 29, 1968, 378-389.

[16] Schober, G.: Semicontinuity of curve functionals. - Arch. Ration. Mech. Anal. 33, 1969, $374-376$.

[17] SchUR, I.: Ein Satz über quadratische Formen mit komplexen Koeffizienten. - Amer. J. Math. $67,1945,472-480$.

[18] SHEn, Y.: Pull-back operators by quasisymmetric functions and invariant metrics on Teichmüller spaces. - Complex Var. Theory Appl. 42, 2000, 289-307.

[19] SHEn, Y.: Notes on pull-back operators by quasisymmetric homeomorphisms with applications to Schober's functionals. - Chinese Ann. Math. Ser. A 24, 2003, 209-218; English transl. in Chinese J. Contemp. Math. 24, 2003, 187-196.

[20] SHen, Y.: Faber polynomials with applications to univalent functions with quasiconformal extensions. - Sci. China Ser. A 52, 2009, 2121-2131.

[21] SHEN, Y.: Generalized Fourier coefficients of a quasi-symmetric homeomorphism and Fredholm eigenvalue. - J. Anal. Math. (to appear).

[22] Springer, G.: Fredholm eigenvalues and quasiconformal mapping. - Acta Math. 111, 1964, $121-141$.

Received 27 October 2009 